\title{
Iris Recognition: An Entropy-Based Coding Strategy Robust to Noisy Imaging Environments
}

\author{
Hugo Proença and Luís A. Alexandre \\ Universidade da Beira Interior, IT - Inst. Telecom. \\ UBI, R. Marquês D’Ávila e Bolama, 6200-001, Covilhã, Portugal \\ \{hugomcp, lfbaa\}@di.ubi.pt
}

\begin{abstract}
The iris is currently accepted as one of the most accurate traits for biometric purposes. However, for the sake of accuracy, iris recognition systems rely on good quality images and significantly deteriorate their results when images contain large noisy regions, either due to iris obstructions (eyelids or eyelashes) or reflections (specular or lighting). In this paper we propose an entropy-based iris coding strategy that constructs an unidimensional signal from overlapped angular patches of normalized iris images. Further, in the comparison between biometric signatures we exclusively take into account signatures' segments of varying dimension. The hope is to avoid the comparison between components corrupted by noise and achieve accurate recognition, even on highly noisy images. Our experiments were performed in three widely used iris image databases (third version of CASIA, ICE and UBIRIS) and led us to observe that our proposal significantly decreases the error rates in the recognition of noisy iris images.
\end{abstract}

\section{Introduction}

Continuous efforts have been made in searching for robust and effective iris coding methods, since Daugman's pioneering work on iris recognition was published. Iris recognition has been successfully applied in such distinct domains as airport checkin or refugee control. However, for the sake of accuracy, current systems require that subjects stand close (less than two meters) to the imaging camera and look for a period of about three seconds until the data is captured. This cooperative behavior is indispensable to capture images with enough quality to the recognition task. Simultaneously, it restricts the range of domains where iris recognition can be applied, namely within heterogeneous lighting conditions or under natural lighting environments. In this context, the overcome of these imaging constrains has motivated the efforts of several authors and deserves growing attention from the research community.

Although some of the published iris recognition algorithms perform a noise detection stage and produce a binary mask - used to avoid that noisy components of the biometric signatures are taken into account - we believe that highly heterogeneous lighting environments (specially under natural light) lead to the appearance of regions which, even for humans, are very difficult to classify as "noisy" or "noise-free". Figure 1 illustrates some of the noise factors that result of less constrained image capturing environments. In figure $1 \mathrm{~b}$ large iris regions obstructed by reflections (lighting and specular) can be observed, some of them very difficult to distinguish from the noise-free ones. 


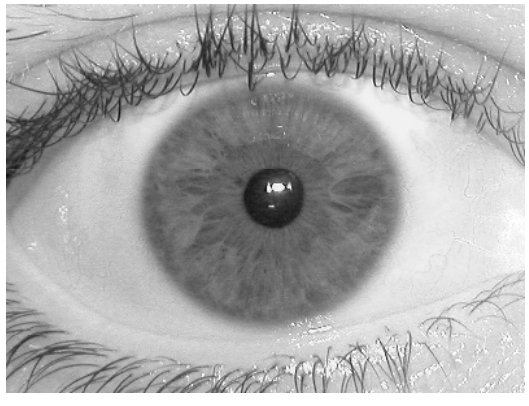

(a) Iris image with good quality.

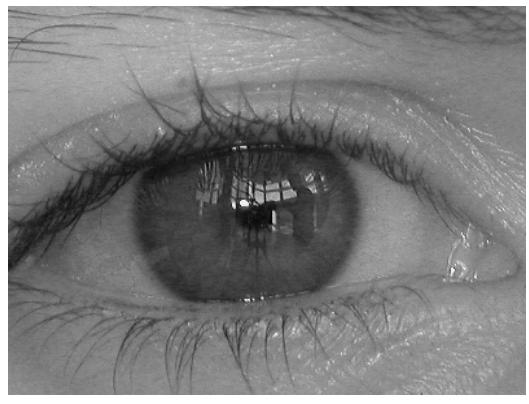

(b) Noisy iris image.

Fig. 1. Comparison between a good quality image and a noise-corrupted one. Figure 1a was captured under high constrained imaging conditions and is completely noise-free. Oppositely, figure $1 \mathrm{~b}$ incorporates several types of noise, resultant from less constrained imaging conditions. It can be observed several iris obstructions - due to eyelids and eyelashes - and large regions of the iris corrupted by reflections, either lighting or specular.

In this paper our main goal is to propose an iris coding and comparison strategy with high robustness to noise. We start by measuring the entropy of consecutive and overlapped angular patches of normalized iris images. This gives an unidimensional signal that contains enough information to distinguish between individuals and, for this reason, is used as biometric signature. Further, in the comparison between signals we take into account shifted segments of varying dimension. The rationale is to profit the portions of the signals that were extracted from noise-free regions and hope that they contain enough information to reliably perform recognition.

Our experiments were performed in three iris image databases with different amounts of noise (third version of CASIA [1], ICE [2] and UBIRIS [3]). For comparison, we selected three of the most cited iris recognition algorithms (Daugman's [4], Wildes' [5] and Tan et al.'s [6]), that we believe to represent the majority of the published approaches. As described in the results' section, although the proposed method obtained roughly similar results to the other algorithms in the less noisy data sets (CASIA and ICE), it achieved smaller error rates in the recognition of the highly noisy iris images of the UBIRIS database. However, it should be stressed that some of this improvement was obtained at the expenses of a significant increase in the computational requirements of our proposal, since its computation time was about the double of the compared algorithms.

The remaining of this paper is organized as follows: section 2 briefly summarizes the most cited iris recognition methods. A detailed description of the proposed feature extraction and comparison method is given in section 3. Section 4 reports the experiments and discusses the results and, finally, section 5 presents the conclusions and points some directions for our further work. 


\section{Iris Recognition}

Figure 2 illustrates the typical stages of iris recognition systems, which, in spite of the specificities of the different proposals, share the given structure. The initial stage deals with iris segmentation. This process consists in localize the iris inner (pupillary) and outer (scleric) borders, assuming either circular or elliptical shapes for both of the borders. In 1993, J. Daugman [4] proposed an integro-differential operator to find both the iris inner and outer borders. Similarly, [7] proposed integro-differential operators that search over the $\mathbb{N}^{3}$ space, with the goal of maximizing the equations that identify the iris borders. Wildes [5] achieved iris segmentation through a gradient based binary edge map construction followed by circular Hough transform. In [8], the authors proposed a method based in Wildes' method, that, together with a clustering process, achieves robustness for non-cooperative imaging environments.

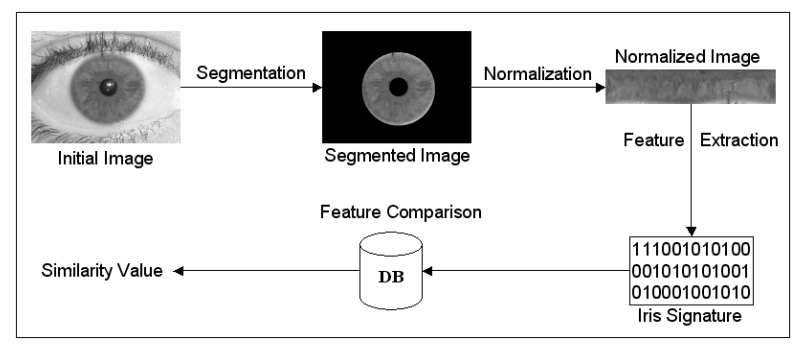

Fig. 2. Typical stages of iris recognition systems.

In order to compensate variations in the pupils size and in the image capturing distances, it is usual to translate the segmented iris data into a fixed length and dimensionless polar coordinate system. This stage is usually accomplished through the method proposed by Daugman [9].

Regarding feature extraction, iris recognition approaches can be divided into three major categories: phase-based methods (e.g., [4]), zero-crossing methods (e.g., [10]) and texture-analysis based methods (e.g., [5]). Daugman [4] used multiscale quadrature wavelets to extract texture phase information and obtain an iris signature with 2048 binary components. Boles and Boashash [10] computed the zero-crossing representation of a 1D wavelet at different resolutions of concentric circles. Wildes [5] proposed the characterization of the iris texture through a Laplacian pyramid with 4 different levels (scales).

Lastly, the comparison between iris signatures is performed, producing a numeric dissimilarity value. If this value is higher than a threshold, the system outputs a nonmatch, meaning that each signature belongs to different irises. Otherwise, the system outputs a match, meaning that both signatures were extracted from the same iris. In this stage, it is common to apply different distance metrics (Hamming [4], Euclidean [11], Weighted Euclidean [12]) or methods based on signal correlation [5]. 


\section{Proposed Recognition Method}

As observed by Ma et al. [13], iris features tend to run in the radial direction, meaning that the most valuable information can be found by examining variations in the angular direction of the iris. Also, as reported by Tisse et al. [14], independently of the pupil dilation, the small-scale radial features of the iris remain stable.

Since the work of Shannon [15], the measuring of entropy has been widely used in the information theory domain and, more particularly, in image processing. Its use was recently reported for several purposes, namely to describe the visual information of images (e.g., [16] and [17]). It is defined as the average number of binary symbols necessary to code an input, given the probability of that input appearing an a stream. High entropy is associated with a high variance in the pixel values, while low entropy indicates that the pixel values are fairly uniform, and hence little detail can be derived from them. The information contained in an image can be regarded as mathematically identical to negative entropy. If any possible intensity value is likely to be next to any other there would be no information present. In this context, the probabilities of which pixel is going to be next to which other gives the information.

Let $I$ be a grayscale image quantized to $l$ intensity levels, the image entropy $h()$ : $\mathbb{N}^{N \times N} \rightarrow \mathbb{R}^{+}$is given by

$$
h(I)=-\sum_{i=1}^{l} p_{i}(I) \log _{2}\left(p_{i}(I)\right)
$$

where $p_{i}$ is the probability of the $i^{\text {th }}$ quantized intensity level in the image $I$

$$
p_{i}(I)=\frac{1}{r \times c} \sum_{r^{\prime}=1}^{r} \sum_{c^{\prime}=1}^{c} \mathbb{I}_{\left\{I\left(r^{\prime}, c^{\prime}\right) \in\left[\text { inf }_{i}, \text { sup }_{i}\right]\right\}}
$$

where $r$ and $c$ are the number of columns and rows of the image, $I\left(r^{\prime}, c^{\prime}\right)$ is the intensity value of the pixel $\left(r^{\prime}, c^{\prime}\right),\left[i n f_{i}\right.$, sup $\left._{i}\right]$ is the interval of intensities of the $i^{t} h$ quantized level and $\mathbb{I}_{\{.\}}$is the characteristic function.

\subsection{Feature Extraction}

As illustrated by figure 3, our feature extraction strategy measures the entropy of overlapped angular windows of the normalized iris images. The goal is to construct an unidimensional signal $s$ that contains information about the variations in the image entropy across the angular iris direction, which is believed to provide the most valuable discriminating biometric information.

Let $N$ be a segmented and normalized iris image, with $r_{N}$ rows and $c_{N}$ columns $\left(r_{N} \times c_{N}\right)$. Also, let $W_{i}$ be an $r_{N} \times\left(c_{W}+1\right)$ image window, centered in the $i^{t h}$ column of $N$ and composed by the columns $\left\{i-\frac{c_{W}}{2}, \ldots, i+\frac{c_{W}}{2}\right\}$ of $N$. The extracted signal $s=\left\{s_{1}, \ldots, s_{c_{N}}\right\}$ contains $c_{N}$ components given by

$$
s_{i}=h\left(W_{i}\right)
$$




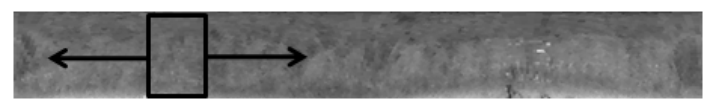

Fig. 3. Proposed feature extraction strategy. We construct an unidimensional signal through the measuring of the entropy of overlapped angular patches of normalized iris images. Each component of the signal $s$ is given by the image entropy of the image window centered at the $i$ column, spanned over all the rows of the normalized image and with fixed width.

Figure 4 contains examples of signals extracted from three normalized iris images. Figures $4 \mathrm{a}$ and $4 \mathrm{~b}$ respectively illustrate signals extracted from a noise-free and a noisy image of the same iris. Here, the high similarity between the first 150 components of both signals can be observed. These components were extracted from the noise-free regions of the noisy iris image. Figure $4 \mathrm{c}$ represents a signal extracted from a different iris and the differences regarding any of the other signals are evident.

As described in the next sub-section, the main challenge is to reliably conclude about the subjects' identity independently of the signal variations that result from different imaging environments and noisy imaging conditions. This is the goal of our feature comparison method.

\subsection{Feature Comparison}

The underlaying idea of our proposed feature comparison method was published in [18] and consists in the direct correspondence between the number of compared components of the biometric signatures and the dissimilarity threshold that distinguish between matches and non-matches. The goal is to perform recognition of an individual using exclusively small portions of its biometric signature, those that are not corrupted by any type of noise.

In the following discussion, we will use a superscript to distinguish between signals extracted from different iris images, such as $s^{1}$ and $s^{2}$. Also, $s^{1}(a, b)$ denotes the shifted segment ( $a$ positions) of the signal $s^{1}$ with $b$ components, such that $s^{1}(a, b)=$ $\left\{s_{a}, \ldots, s_{(a+b) \bmod c_{N}}\right\}$.

The function $t(x): \mathbb{N} \rightarrow \mathbb{R}$ gives the threshold that distinguishes between match and non-match comparisons for signatures with $x$ components

$$
t(x)=\frac{\left(M_{d}-m_{d}\right)\left(x-m_{l}\right)}{M_{l}-m_{l}}+m_{d}
$$

where $m_{l}$ and $M_{l}$ are respectively the minimum and maximum number of components of the comparable signals and $m_{d}$ and $M_{d}$ are the minimum and maximum threshold values, $m_{l}<M_{l}$ and $m_{d}<M_{d}$.

The function $f c\left(s^{1}, s^{2}\right)$ performs the comparison between segments of $s^{1}$ and $s^{2}$ and produces the decision about the identity of the subjects from where the signals were extracted:

$$
f c\left(s^{1}, s^{2}\right)= \begin{cases}\text { Match } & d\left(s^{1}\left(a_{i}, b_{j}\right), s^{2}\left(a_{i}, b_{j}\right)\right) \leq t\left(b_{j}\right) \\ \text { Non }- \text { Match }, & \text { otherwise }\end{cases}
$$




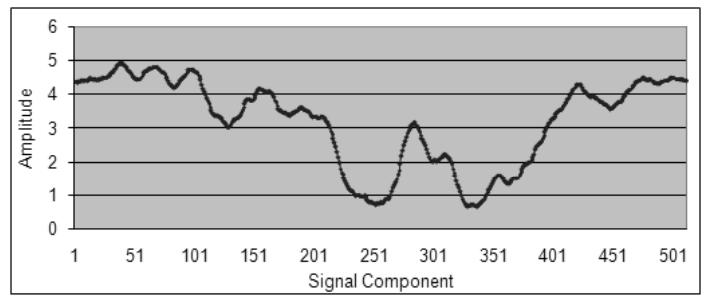

(a) Signal extracted from a noise-free image of subject 1 .

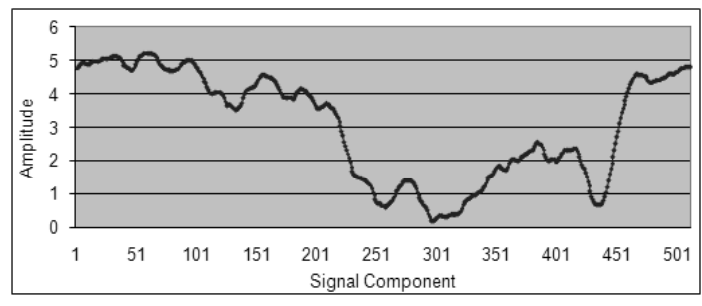

(b) Signal extracted from a noisy image of subject 1 .

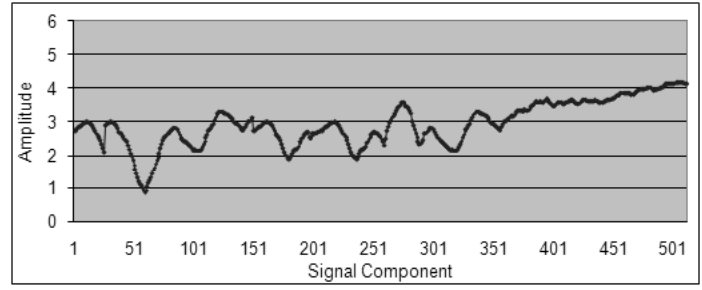

(c) Signal extracted from subject 2 .

Fig. 4. Examples of the unidimensional extracted signals. Figures $4 \mathrm{a}$ and $4 \mathrm{~b}$ contain signals extracted from different images of the same iris, respectively from a noise-free (figure 4a) and a noisy (figure 4b) image. It is notorious the similarity between the first 150 components of the signals, which were extracted from the noise-free regions on both images. Also, the difference between both signals and the illustrated in figure $4 \mathrm{c}$ (extracted from an image of a different iris) is evident.

where $m_{l} \leq a_{i}, b_{j} \leq M_{l}$ and $d()$ is the Euclidean distance.

\section{Experiments and Discussion}

To enable the test of the proposed recognition method, we analyzed the available iris image databases and selected the most appropriate for our purposes. In the following 
we briefly describe the available public iris image databases and the data sets choused for our experiments.

\subsection{Iris Databases}

There are presently seven public and freely available iris image databases for biometric purposes: Chinese Academy of Sciences [1] (CASIA, three distinct versions), Multimedia University (MMU), University of Bath (BATH), University of Olomuc (UPOL), Iris Challenge Evaluation [2] (ICE), West Virginia University (WVU) and University of Beira Interior [3] (UBIRIS).

CASIA database is by far the most widely used for iris biometric purposes and has three distinct versions. However, its images incorporate few types of noise, almost exclusively related with eyelid and eyelash obstructions, similarly to the images of the MMU and BATH databases. UPOL images were captured with an optometric framework, obtaining optimal images with extremely similar characteristics. Although ICE and WVU databases contain images with more noise factors, their lack of images with significant reflections within the iris rings constitutes a weak point, regarding the simulation of less constrained imaging conditions. Oppositely, images of the UBIRIS database were captured under natural lighting and heterogenous imaging conditions, which explains their higher heterogeneity.

Due to this, we selected 800 images from 80 subjects of the UBIRIS database. In order to evaluate the recognition accuracy both in highly and less noisy environments, an equal number of images from the CASIA (third version) and ICE databases were selected. Further, we divided each data set into two halves. The first data sets $U B I R I S_{t r}, C A S I A_{t r}$ and $I C E_{t r}$ - were used as training sets and the later - UBIRIS $S_{t t}$, $C A S I A_{t t}$ and $I C E_{t t}$ - to evaluate the recognition accuracy.

Each data set enables respectively 1800 and 78000 intra- and inter-class comparisons. Images of the UBIRIS data sets contain iris obstructions by eyelids and eyelashes, poor focused and motion blurred irises and irises with specular and lighting reflections, while those of the ICE data sets contain iris obstructions by eyelids and eyelashes, offangle iris images and eyes with contact lenses. Images of the CASIA data sets have minor portions of noise, almost exclusively related with iris obstructions by eyelids and eyelashes.

\subsection{Recognition Methods Used as Comparison}

As above stated, we compared the results obtained by the proposed recognition method and three of the most cited iris recognition algorithms (Daugman [9], Wildes [5] and Tan et al [6]), which we believe to represent the majority of the published proposals. Also, the algorithm proposed by Daugman is the basis of all the commercially deployed recognition systems.

\subsection{Results and Discussion}

For all images of the above described data sets we extracted the biometric signatures, according to each above mentioned approach. Further, we performed the feature comparison with all the remaining signatures of the same data set. In order to avoid that 


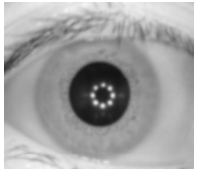

(a) Poor focused iris image.

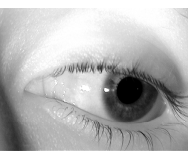

(d) Off-angle iris image.

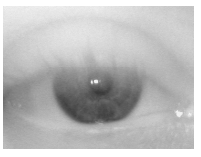

(g) Motion blurred iris im-

age.

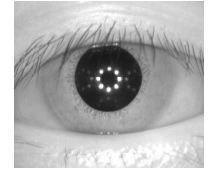

(b) Iris with eyelid obstruc-

tions.

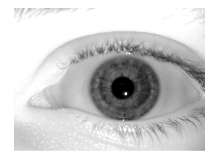

(e) Eye with contact lenses.

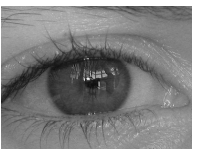

(h) Iris with large reflection areas.

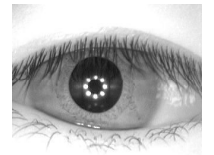

(c) Iris with eyelid and eyelash obstructions.

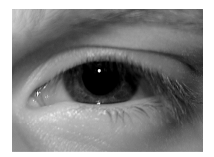

(f) Iris with extreme eyelid and eyelash obstructions.

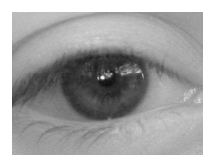

(i) Iris with large reflection areas and eyelids and eyelash

Fig. 5. Examples of the images used in our experiments. The first row contains images of the third version of the $C A S I A$ database, which commonly have iris obstructions due to eyelids or eyelashes and poor focused images. The second row contains images of the $I C E$ database. It incorporates off-angle images, iris obstructions and several eyes with contact lenses. Finally, the third row exemplifies some of the highly noisy images contained by the $U B I R I S$ database. This database contains, apart from the above referred types of noise, images with large reflection regions, either specular or lighting.

inaccuracies in the iris segmentation and normalization stages corrupt the obtained results, we manually verified the accuracy of the iris segmentation algorithms.

Figure 6 compares the obtained receiver operating curves (ROCs) by our proposal (continuous lines with circular data points), Daugman's (dashed lines with triangular data points), Wildes' (dotted lines with cross data points) and Tan et al.'s (large dashed lines with square data points) in the $U B I R I S_{t t}$ (figure 6a), $I C E_{t t}$ (figure 6b) and $C A S I A_{t t}$ (figure 6c) data sets. It can be observed that the error rates obtained in the less noisy data sets were similar to those obtained by the Wildes and Tan et al. algorithms. In both these data sets, Daugman's recognition algorithm achieved better results. However, in the highly noisy images of the $U B I R I S_{t t}$ data set, which incorporates images with very large reflection regions, our proposal outperformed the results obtained by any of the other recognition algorithms, which confirms its higher robustness to noise. 


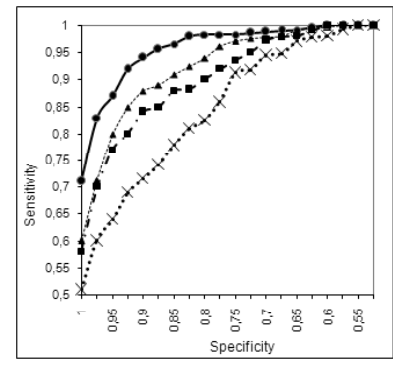

(a) ROCs obtained in the UBIRIStt data set

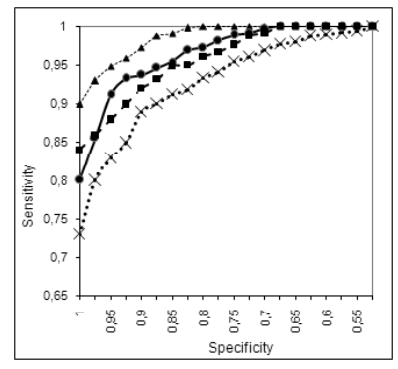

(b) ROCs obtained in the $I C E_{t t}$ data set

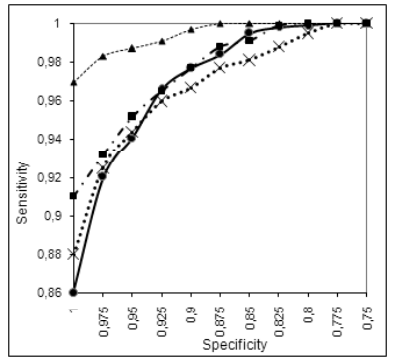

(c) ROCs obtained in the CASI $A_{t t}$ data set

Fig. 6. Comparison between the ROCs obtained in the $U B I R I S_{t t}$ (figure 6a), $I C E_{t t}$ (figure 6b) and $C A S I A_{t t}$ (figure 6c) data sets. The continuous lines with circular data points represent our proposal. Daugman's is represented by the dashed lines with triangular data points and Wildes' by the dotted lines with cross data points. Finally, the large dashed lines with square data points represents the recognition method proposed by Tan et al.. Although our proposal obtained higher error rates than the other methods used as comparison in the recognition of images of the $C A S I A$ and $I C E$ data sets, it outperforms these methods in the recognition of highly noisy images (figure 6a).

Table 1. Comparison between the error rates obtained by the tested algorithms in the images from the $U B I R I S_{t t}, I C E_{t t}$ and $C A S I A_{t t}$ data sets.

\begin{tabular}{|l|c|c|c|}
\hline $\begin{array}{l}\text { Recognition } \\
\text { method }\end{array}$ & FRR, FAR=0 (\%) & EER (\%) & FR \\
\hline$U B I R I S_{t t}$ data set \\
\hline Proposed & $29.17 \pm 0.04$ & $08.91 \pm 0.02$ & 86.17 \\
\hline Daugman & $40.02 \pm 0.06$ & $11.47 \pm 0.03$ & 75.83 \\
\hline Wildes & $49.73 \pm 0.07$ & $18.73 \pm 0.03$ & 54.19 \\
\hline Tan et al. & $42.77 \pm 0.06$ & $13.70 \pm 0.03$ & 70.41 \\
\hline$I C E_{t t}$ data set \\
\hline Proposed & $20.05 \pm 0.03$ & $06.70 \pm 0.02$ & 109.14 \\
\hline Daugman & $11.30 \pm 0.03$ & $04.82 \pm 0.02$ & 143.19 \\
\hline Wildes & $27.11 \pm 0.04$ & $10.58 \pm 0.03$ & 105.12 \\
\hline Tan et al. & $16.06 \pm 0.03$ & $08.86 \pm 0.02$ & 127.95 \\
\hline$C A S I A_{t t}$ data set & $14.03 \pm 0.03$ & $05.03 \pm 0.02$ & 119.72 \\
\hline Proposed & $03.97 \pm 0.01$ & $01.90 \pm 0.01$ & 147.16 \\
\hline Daugman & $12.09 \pm 0.03$ & $05.25 \pm 0.02$ & 105.91 \\
\hline Wildes & $09.27 \pm 0.02$ & $04.94 \pm 0.02$ & 127.93 \\
\hline Tan et al. &
\end{tabular}

Table 1 summarizes the error rates obtained by the above described algorithms in the $U B I R I S_{t t}, I C E_{t t}$ and $C A S I A_{t t}$ data sets. The first column identifies the classification method, the second contains the false rejection rates when preventing the false 
accept errors $(F R R, F A R=0)$. EER corresponds to the approximated equal error rate and, finally, the last column contains the value of a Fisher-ratio test (FR) given by:

$$
F R=\frac{\left(\mu^{E}-\mu^{I}\right)^{2}}{\frac{\sigma^{2}}{N^{I}}+\frac{\sigma^{E^{2}}}{N^{E}}}
$$

where $\mu^{I}$ and $\mu^{E}$ respectively indicate the mean of the intra- and inter-class dissimilarities. $\sigma^{I}$ and $\sigma^{E}$ indicate the respective standard deviations and $N^{I}$ and $N^{E}$ are, respectively, the number of intra- and inter-class comparisons. All the error rates are expressed for a confidence interval of $95 \%$.

Once again, it can be observed that Daugman's recognition method achieved higher separability between the intra- and inter-class comparisons in the less noisy data sets $\left(C A S I A_{t t}\right.$ and $\left.I C E_{t t}\right)$. In both cases, the values of the FR test are higher and the error rates (either the $E E R$ or the $F R R, F A R=0$ ) smaller. However, when images incorporate large reflection regions, all the algorithms used in the comparison with our proposal significantly decreased their accuracy, showing their small robustness to noise. In the $U B I R I S_{t t}$ data set the proposed recognition method achieved significantly lower error rates.

The high robustness of our proposal can be highlighted by the proportion values between the results obtained in the noisiest and less noisy iris image data sets: $\left(\frac{U B I R I S_{t t}}{C A S I A_{t t}}\right)$ and $\left(\frac{U B I R I S_{t t}}{I C E_{t t}}\right)$. For the EER we obtained the proportion values of respectively 1.77 and 1.32, which are significantly lower than those obtained for the Daugman's (6.03 and 2.37), Wildes' (3.56 and 1.77) and Tan et al.'s (2.77 and 1.54) recognition algorithms. Similar values were obtained for the other error $(F R R, F A R=0)$ and separability $(F R)$ measures.

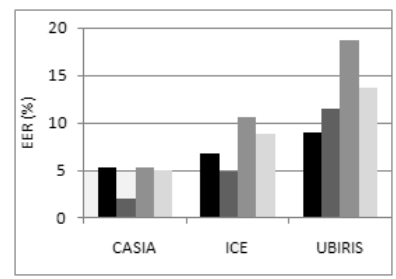

(a) Obtained EER in the three data sets.

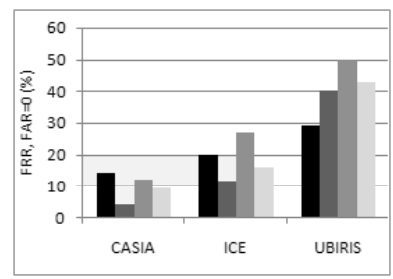

(b) Obtained $F R R, F A R=0$ in the three data sets.

Fig. 7. Degradation in the accuracy of the tested algorithms, regarding the amount of noise of the data sets. The $U B I R I S$ is the noisiest and $C A S I A$ the less noisy. Our proposal, Daugman's, Wildes' and Tan et al.'s are respectively represented by the darkest to the brightest bar series. Although our proposal obtained higher error rates in the less noisy data sets (CASIA and ICE), a smaller degradation in the results is evident.

Figure 7 illustrates the increase of the error rates (EER in figure 7a and FRR, FAR=0 in figure $7 \mathrm{~b}$ ) obtained by the experimented algorithms, regarding the amount of noise 
in the iris images. The vertical axes contains the error rates (\%) and the horizontal contain the data sets where these errors were obtained, ordered from the less noisy to the noisiest data sets. Our proposal, Daugman's, Wildes' and Tan et al.'s are respectively represented by the darkest to the brightest bar series.

\section{Computation Time}

The algorithms were implemented in $\mathrm{C}++$, following an object-oriented paradigm and running in an image-processing framework developed by the authors. This framework is not optimized for execution speed, as the algorithm's implementation was made without these concerns, but instead with a user-friendly objective. However, the proportion between the average execution time of the implemented algorithms can be evaluated and assumptions about their optimized execution time can be made. The fastest recognition algorithm was the one of Wildes, with average execution time of about 1.6 seconds. The computation time observed for the Daugman's and Ma et al. algorithms was about 17 and $21 \%$ higher. Finally, our recognition proposal has higher computational requirements, essentially due to the feature comparison stage. The observed average execution time was more than $90 \%$ higher than the one of Wildes.

We are focused on the development and evaluation of alternate feature comparison strategies that present similar robustness to noisy signals and have smaller computational requirements.

\section{Conclusions and Further Work}

Having observed the significant impact of noise in the accuracy of the most relevant iris recognition algorithms, we described a new iris recognition strategy more robust to noise. The proposed method encodes the iris information into an unidimensional signal, that measures the entropy of overlapped iris angular patches. For the purpose of signature matching, we compared segments of the signals with varying dimension, hoping that noise-free components allow reliable biometric recognition. Here, the dissimilarity threshold that distinguishes between match and non-match comparisons has direct correspondence with the length of the compared signal segments.

Although our proposal did not outperform the compared ones in the less noisy data sets (CASIA and ICE), we observed a significantly minor degradation in the recognition of the highly noisy images of the $U B I R I S$ database. This makes our proposal more suitable for the application within less constrained imaging environments.

However, the computational requirements of the proposed method are a concern. Our efforts are presently concentrated in decrease the computational complexity, as well in the search of alternate feature comparison methods more accurate in the recognition of noise-free images.

\section{Acknowledgements}

We acknowledge the support of the Portuguese Fundação para a Ciência e Tecnologia (FCT) and FEDER, project PTDC/EIA/69106/2006. 


\section{References}

1. Institute of Automation, Chinese Academy of Sciences: CASIA iris image database (2004) http://www.sinobiometrics.com.

2. National Institute of Standards and Technology: Iris challenge evaluation (2006) http://iris.nist.gov/ICE/.

3. Proença, H., Alexandre, L.A.: UBIRIS: A noisy iris image database. In: Proceedings of the $13^{\text {th }}$ International Conference on Image Analysis and Processing (ICIAP2005), Calgary (2005) 970-977 http://iris.di.ubi.pt.

4. Daugman, J.G.: High confidence visual recognition of persons by a test of statistical independence. IEEE Transactions on Pattern Analysis and Machine Intelligence, vol. 25, no. 11 (1993) 1148-1161

5. Wildes, R.P.: Iris recognition: an emerging biometric technology. In: Proceedings of the IEEE, vol. 85, no.9, U.S.A. (1997) 1348-1363

6. Ma, L., Tan, T., Zhang, D., Wang, Y.: Local intensity variation analysis for iris recognition. Pattern recognition, vol. 37, no. 6 (2004) 1287-1298

7. Camus, T., Wildes, R.: Reliable and fast eye finding in close-up images. In: Proceedings of the IEEE 16th International Conference on Pattern Recognition, Quebec (2002) 389-394

8. Proença, H., Alexandre, L.A.: Iris segmentation methodology for non-cooperative iris recognition. IEE Proc. Vision, Image \& Signal Processing, vol. 153, issue 2 (2006) 199-205

9. Daugman, J.G.: How iris recognition works. IEEE Transactions on Circuits and Systems for Video Technology, vol. 14, no. 1 (2004) 21-30

10. Boles, W.W., Boashash, B.: A human identification technique using images of the iris and wavelet transform. IEEE Transactions on Signal Processing, vol. 46, no. 4 (1998) 1185-1188

11. Huang, Y., Luo, S., Chen, E.: An efficient iris recognition system. In: Proceedings of the First International Conference on Machine Learning and Cybernetics, China (2002) 450-454

12. Ma, L., Wang, Y., Tan, T.: Iris recognition using circular symmetric filters. In: Proceedings of the $25^{\text {th }}$ International Conference on Pattern Recognition (ICPR02), vol. 2, Quebec (2002) 414-417

13. Ma, L., Tan, T., Wang, Y., Zhang, D.: Personal identification based on iris texture analysis. IEEE Transactions on Pattern Analysis and Machine Intelligence, vol. 25, no. 12 (2003) 2519-2533

14. Tisse, C., Martin, L., Torres, L., Robert, M.: Person identification technique using human iris recognition. In: Proceedings of the 25th International Conference on Vision Interface, Calgary (2002) 294-299

15. Shannon, C.E.: A mathematical theory of communication. Bell System Technical Journal, vol. 27 (1948) 623-656

16. Ferraro, M., Boccignone, G., Caelli, T.: Entropy-based representation of image information. Pattern Recognition Letters, vol. 23 (2002) 1391-1398

17. Yanai, K., Barnard, K.: Image region entropy: a measure of "visualness" of web images associated with one concept. In: Proceedings of the $13^{\text {th }}$ annual ACM International Conference on Multimedia, Singapore (2005) 419-422

18. Proença, H., Alexandre, L.A.: Toward non-cooperative iris recognition: A classification approach using multiple signatures. IEEE Transactions on Pattern Analysis and Machine Inteligence, vol. 9, no.4 (2007) 607-612 